Surface Science 96 (1980) 482-507

(c) North-Holland Publishing Company

\title{
ELLIPSOMETRY OF CLEAN SURFACES, SUBMONOLAYER AND MONOLAYER FILMS
}

\author{
F.H.P.M. HABRAKEN, O.L.J. GIJZEMAN and G.A. BOOTSMA * \\ Van 't Hoff Laboratory, University of Utrecht, Padualaan 8, 3584 CH Utrecht. The Netherlands
}

Received 20 August 1979

The geometric and electronic structure of the surface region of a crystal is often different from the bulk structure and thereforc the optical properties differ in principle also. Theorics for the optical properties of (sub)monolayer films are compared, with special attention to anisotropic layers. The review of experimental studies in ultra high vacuum systems mostly concerns ellipsometric measurements of chemisorption of oxygen on metal surfaces $(\mathrm{Ag}, \mathrm{Cu}, \mathrm{Ni})$. With the combination ellipsometry-AES-LEED linear relationships have been observed between the change in $\Delta$ and the coverage of oxygen chemisorbed on $\mathrm{Ag}(110), \mathrm{Cu}(100)$, $\mathrm{Cu}(111)$ and $\mathrm{Cu}(110)$. At room temperature the $\delta \Delta$ values per oxygen atom on the different $\mathrm{Cu}$ planes are equal, and are larger than the $\delta \Delta$ value per atom taken up in a later stage of oxidation. For the anisotropic $\mathrm{Cu}(110)$ plane the $\delta \Delta$ and $\delta \psi$ values per adsorbed oxygen atom depend on the crystal temperature and the azimuthal orientation of the plane of incidence of the light beam. In order to explain the observed changes in $\Delta$ and $\psi$, models are discussed in which changes in the substrate, caused by chemisorption, are taken into account.

\section{Introduction}

The availability of ultra high vacuum (UHV) and sensitive surface analytical techniques makes it possible to tackle again fundamental problems, such as those studied by the founders of ellipsometry, Drude [1] and Rayleigh [2]. Their original question: do chemically clean surfaces show ellipticity at the Brewsterian angle due to "very powerful forces of whose action we know but little" $[2,3]$ still remains unanswered. Also the applicability of ellipsometry to fields like passivity and corrosion of metals, adsorption, heterogeneous catalysis and surface migration (mentioned by Tronstad [4]) has been improved by automation of the measurements.

Since the study of oxygen adsorption on a clean silicon surface in UHV by Archer and Gobeli [5], a number of ellipsometric studies of adsorption in (sub)monolayer quantities have been performed, notably on semiconductors (for reviews see refs. $[6,7])$. For physisorption, where the interaction between adsorbate

* Present address: Department of Applied Physics, Twente University of Technology, 7500 AE Enschede, The Netherlands. 
and substrate is weak, e.g. krypton on silicon and germanium $[7,8]$ and xenon on graphite $[9,10]$, the ellipsometric results could be interpreted with a simple extrapolated macroscopic theory as well as with microscopic approaches, originally presented by Strachan [11] and Sivukhin [12,13]. For chemisorption on semiconductors changes in the optical properties of the substrate induced by the adsorbed layer had to be taken into account [6-8].

In the next section the previous review of theories for the optical properties of (sub)monolayer films [6] will be extended, with special attention to anisotropic layers. The review of experimental studies in UHV systems will mostly concern ellipsometric measurements of the chemisorption of oxygen on metal surfaces $(\mathrm{Ag}$, $\mathrm{Cu}, \mathrm{Ni})$. In order to explain the experimental results models will be discussed in which changes in the substrate have been considered.

\section{Theories for the (sub)monolayer range}

Theories for the (sub)monolayer range may be classified in two groups. The most basic approach is to calculate the scattering amplitude of an adsorbed molecule, as modified by the substrate, from quantum mechanical principles. This necessitates a complete quantum treatment of the radiation field. The final, outgoing, photon state is then obtained by summing the individual contributions with the proper phase factors $[14,15]$.

A second approach utilizes the macroscopic Maxwell equations for a system of stratified layers. Within this framework all properties of the adlayer are accounted for in terms of $\epsilon$, its dielectric constant. This constant may be obtained from quantum mechanical considerations as well, by calculating the modification of the substrate band structure due to the adsorbate [16]. A refinement of this procedure will involve the calculation of the spatial dispersion of $\epsilon$ [17].

In practice, the determination of the dielectric constant is usually done backwards, i.e. from observed changes in the light intensity and state of polarization upon reflection, one tries to calculate $\epsilon$ for a layer with an assumed thickness or the integral of $\epsilon$ over an unspecified transition layer $[18,19]$. In this section we will try to outline theories for the dielectric constant of a (sub)monolayer and its relation to basic molecular properties.

\subsection{Stratified-layer model with biaxial anisotropy}

We start with the classical stratified-layer model with plane-parallel boundaries for the system ambient/adsorbed layer/substrate (fig. 1). The dielectric constant $\epsilon$ and index of refraction $\tilde{n}$ of substrate and layer may both be complex:

$\epsilon=\epsilon^{\prime}-\mathrm{i} \epsilon^{\prime \prime}$ and $\tilde{n}=n-\mathrm{i} k=n(1-\mathrm{i} \kappa)$, with $\epsilon=\widetilde{n}^{2}$.

The substrate is considered to be isotropic, whereas the layer may be biaxially 


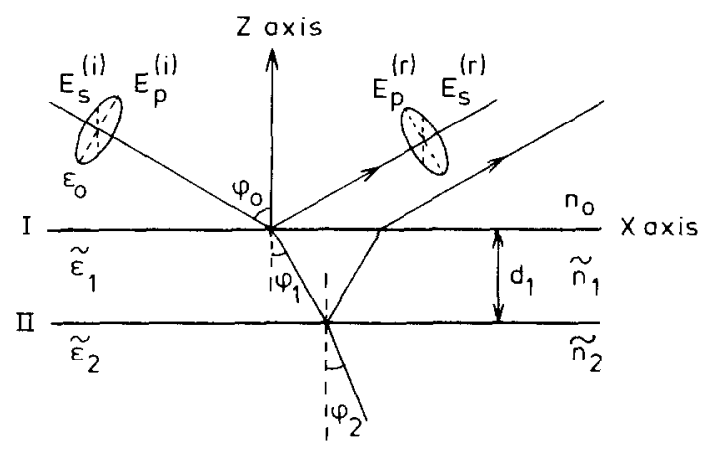

Fig. 1. Reflection of polarized light at a substrate covered with one layer.

anisotropic with principal indices of refraction $\widetilde{n}_{1 x}, \widetilde{n}_{1 y}$ and $\widetilde{n}_{1 z}$.

If two of the principal axes ( $x$ and $y$ ) of the biaxial film are parallel to the phase boundaries I and II, while the third $(z)$ is perpendicular to them (plane of incidence $x z$, fig. 1), we get for the amplitude reflection coefficients $[20,21]$ :

$R_{\mathrm{p}}=\frac{r_{\mathrm{Ip}}+r_{\mathrm{IIp}} \exp \left(-\mathrm{i} 2 \beta_{\mathrm{p}}\right)}{1+r_{\mathrm{Ip}} r_{I I \mathrm{p}} \exp \left(-\mathrm{i} 2 \beta_{\mathrm{p}}\right)}, \quad R_{\mathrm{s}}=\frac{r_{\mathrm{IS}}+r_{\mathrm{IIs}} \exp \left(-\mathrm{i} 2 \beta_{\mathrm{s}}\right)}{1+r_{\mathrm{Is}} r_{\mathrm{IIs}} \exp \left(-\mathrm{i} 2 \beta_{\mathrm{s}}\right)}$.

The Fresnel coefficients for the two interfaces are

$r_{\text {Ip }}=\frac{\widetilde{n}_{1 x} \widetilde{n}_{1 z} \cos \phi_{0}-n_{0}\left(\widetilde{n}_{1 z}^{2}-n_{0}^{2} \sin ^{2} \phi_{0}\right)^{1 / 2}}{\widetilde{n}_{1 x} \widetilde{n}_{1 z} \cos \phi_{0}+n_{0}\left(\widetilde{n}_{1 z}^{2}-n_{0}^{2} \sin ^{2} \phi_{0}\right)^{1 / 2}}$

$r_{\mathrm{Is}}=\frac{n_{0} \cos \phi_{0}-\left(\widetilde{n}_{1 y}^{2}-n_{0}^{2} \sin ^{2} \phi_{0}\right)^{1 / 2}}{n_{0} \cos \phi_{0}+\left(\widetilde{n}_{1 y}^{2}-n_{0}^{2} \sin ^{2} \phi_{0}\right)^{1 / 2}}$,

$r_{\text {IIp }}=\frac{\widetilde{n}_{2}\left(\widetilde{n}_{1 z}^{2}-\widetilde{n}_{2}^{2} \sin ^{2} \widetilde{\phi}_{2}\right)^{1 / 2}-\widetilde{n}_{1 x^{n}} \widetilde{n}_{1 z} \cos \widetilde{\phi}_{2}}{\widetilde{n}_{2}\left(\widetilde{n}_{1 z}^{2}-\widetilde{n}_{2}^{2} \sin ^{2} \widetilde{\phi}_{2}\right)^{1 / 2}+\widetilde{n}_{1 x} \widetilde{n}_{1 z} \cos \widetilde{\phi}_{2}}$,

$r_{1 \mathrm{~s}}=\frac{\left(\widetilde{n}_{1 y}^{2}-\widetilde{n}_{2}^{2} \sin ^{2} \widetilde{\phi}_{2}\right)^{1 / 2}-\widetilde{n}_{2} \cos \widetilde{\phi}_{2}}{\left(\widetilde{n}_{1 y}^{2}-\widetilde{n}_{2}^{2} \sin ^{2} \widetilde{\phi}_{2}\right)^{1 / 2}+\widetilde{n}_{2} \cos \widetilde{\phi}_{2}}$.

Furthermore, the phase differences $\beta$ in eq. (2) are given by

$\beta_{\mathfrak{p}}=2 \pi \frac{d_{1}}{\lambda} \frac{\widetilde{n}_{1 x}}{\widetilde{n}_{1 z}}\left(\widetilde{n}_{1 z}^{2}-n_{0}^{2} \sin ^{2} \phi_{0}\right)^{1 / 2}$,

$\beta_{\mathrm{s}}=2 \pi \frac{d_{1}}{\lambda}\left(\widetilde{n}_{1 y}^{2}-n_{0}^{2} \sin ^{2} \phi_{0}\right)^{1 / 2}$.

The angle of incidence, $\phi_{0}$, and angle of refraction in the substrate, $\widetilde{\phi}_{2}$, are related by Snell's law

$n_{0} \sin \phi_{0}=\widetilde{n}_{2} \sin \widetilde{\phi}_{2}$. 
As usual in ellipsometry the effect upon reflection is summarized in the equation

$\frac{R_{\mathrm{p}}}{R_{\mathrm{s}}}=\frac{E_{\mathrm{p}}^{(\mathrm{r})} / E_{\mathrm{p}}^{(\mathrm{i})}}{E_{\mathrm{s}}^{(\mathrm{r})} / E_{\mathrm{s}}^{(\mathrm{i})}}=\tan \psi \exp (\mathrm{i} \Delta)$.

For reflection at the clean substrate, characterized by $\Delta$ and $\Psi$ one gets

$\tan \bar{\psi} \exp (\mathrm{i} \bar{\Delta})=\frac{\tilde{n}_{2} \cos \phi_{0}-n_{0} \cos \widetilde{\phi}_{2}}{\widetilde{n}_{2} \cos \phi_{0}+n_{0} \cos \widetilde{\phi}_{2}} \frac{n_{0} \cos \phi_{0}+\widetilde{n}_{2} \cos \widetilde{\phi}_{2}}{n_{0} \cos \phi_{0}-\widetilde{n}_{2} \cos \widetilde{\phi}_{2}}$.

In the previous review [6] for thin isotropic and non-absorbing thin films the firstorder Drude approximations were quoted for $\tan \psi \exp (\mathrm{i} \Delta)$ and

$\delta \Delta=\bar{\Delta}-\Delta \quad$ and $\quad \delta \psi=\bar{\psi}-\psi$.

For relatively simple cases with anisotropic films, e.g. uniaxial nonabsorbing layers [22], such approximations may be of value for reasons of clarity and comparison. However, because computer calculations with the exact equations (2)-(10) are easily performed, first-order approximations for more general cases of anisotropy will not be presented here.

\subsection{Integral approach}

A more realistic description of the optical properties of an interface region than the stratified-layer model may be given by introducing, in analogy with the thermodynamic treatment of surface regions (the Gibbs or Guggenheim models, see ref. [23]), surface excess functions, in this case for the continuously varying dielectric constants. To get the reflection coefficients the usual boundary conditions have to be replaced by integral boundary conditions. This integral approach, recognized by Drude [1], was recently emphasized again by Plieth et al. [18,19], notably for the description of the optical behaviour of electrode surfaces in electrolyte solutions. For an interface with uniaxial anisotropy four quantities were chosen to characterize the optical response of the system, namely the real and imaginary parts of the integrals

$$
\int\left(\epsilon_{\mathrm{t}}-\epsilon_{\mathrm{ref}}\right) \mathrm{d} z \text { and } \int\left\{\frac{1}{\epsilon_{\mathrm{n}}}-\frac{1}{\epsilon_{\mathrm{ref}}}\right\} \mathrm{d} z .
$$

Here $\epsilon_{\mathrm{t}}$ and $\epsilon_{\mathrm{n}}$ are the tangential and normal component of the dielectric tensor and the integration is carried out from the homogeneous ambient to the homogeneous substrate phase; $\epsilon_{\text {ref }}$ represents the film-free surface (reference state). However, it is common practice to define a mean dielectric function for the interface region of overall thickness $d_{1}$, as [24]

$$
\left\langle\epsilon_{1}\right\rangle=\frac{1}{d_{1}} \int_{0}^{d_{1}} \epsilon_{1}(z) \mathrm{d} z
$$


The expressions for the reflection coefficients etc. are then identical to those given in the previous section, the optical constants being replaced by the averaged functions.

A central problem with submonolayer films is the relation between the macroscopic quantities $(\epsilon, \widetilde{n}, d)$ and the microscopic parameters, i.e. the polarizability $(\alpha)$, diameter $\left(d_{\mathrm{m}}\right)$, cross-sectional area $(c)$ or degree of coverage $\left(\theta=c / c_{\mathrm{m}}\right.$, with $c_{\mathrm{m}}$ cross-section at $\theta=1$ ) of the adsorbed atoms or molecules. Several, not essentially different, proposals to fill this gap between macroscopic theory and atomic description will be discussed in the next sections.

\subsection{Extrapolated macroscopic theory (Drude model)}

In the macroscopic description for an anisotropic dielectric medium the dielectric constants are obtained by combining the following set of equations [25-27]: (i) relation between effective local field $\boldsymbol{E}^{\mathrm{toc}}$ at a molecule and the averaged macioscopic field $\boldsymbol{E}$ and polarization $\boldsymbol{P}$ :

$E_{j}^{\mathrm{loc}}=E_{j}+L_{j} P_{j}$,

where $j=x, y, z$ and $L_{j}$ is a depolarization factor depending on the anisotropy (shape factor);

(ii) definition of the dielectric constant (in cgs units):

$P_{j}=\frac{\left(\epsilon_{j}-1\right)}{4 \pi} E_{j}$;

(iii) relation between $\boldsymbol{P}$ and $\boldsymbol{E}^{10 c}$, definition of polarizability:

$P_{j}=N \alpha_{j} E_{j}^{\mathrm{loc}}$,

where $N$ is the number of molecules per unit volume and $\alpha_{j}$ the $j j$ component of the molecular polarizability tensor whose principal axes are assumed to be parallel to $x, y$ and $z$.

The combination of eqs. (15), (16) and (17) gives

$\epsilon_{j}-1=4 \pi N \alpha_{j} /\left(1-N \alpha_{j} L_{j}\right)$.

The anisotropy in $\epsilon$ can thus be due to anisotropy in the molecular polarizability or to asymmetry in the shape factor. A well-known method (Lorentz method) for the calculation of the depolarization factors is based on the cavity model in a continuous dielectric $[25,26]$. For isotropic molecules $\left(\alpha_{x}=\alpha_{y}=\alpha_{z}=\alpha\right)$ in isotropic media, with $L_{x}=L_{y}=L_{z}=\frac{4}{3} \pi$, one gets with eq. (18) the Lorentz-Lorenz equation $\left(n^{2}-1\right) /\left(n^{2}+2\right)=\frac{4}{3} \pi N \alpha$,

Extrapolation to a submonolayer yields [6]

$\left(n_{\theta}^{2}-1\right) /\left(n_{\theta}^{2}+2\right)=\frac{4}{3} \pi \alpha \theta / c_{\mathrm{m}} d_{\mathrm{m}}$. 
The optical effect of such a layer follows from the equations in section 2.1 for the isotropic case, with $n_{1}=n_{\theta}$ and $d_{1}=d_{\mathrm{m}}$.

In principle, for arbitrary asymmetric shapes of adsorbed molecules, the shape factors of the corresponding cavities can be calculated and thus also the optical constants. For cylindrical cavities, representing the long-chain molecules in ordered layers of lipids, the results have been presented by Den Engelsen [27]. If the electric field is taken either parallel or normal to the cylinder axis, coinciding with the optic axis of the uniaxially anisotropic medium, one gets for the shape factors

$L_{/ /}=4 \pi\left[1-l /\left(D^{2}+l^{2}\right)^{1 / 2}\right], L_{\perp}=2 \pi l /\left(D^{2}+l^{2}\right)^{1 / 2}$.

Here $l$ is the length and $D$ the diameter of the cylinder. Nontetragonal types of anisotropic media could be treated analogously, e.g. by using the depolarization factors derived for general forms of ellipsoids $[28,29]$. Insertion of the shape factors and polarizabilities in eq. (18) yields the optical constants, and use of eqs. (2)-(12) gives finally the ellipsometric effects upon adsorption.

\subsection{Quasi-microscopic theory (Strachan's model)}

In Strachan's description the interfacial layer is represented by a two-dimensional distribution of Hertzian oscillators, characterized by the scattering indices $\sigma_{x}, \sigma_{y}$ and $\sigma_{z}[11,6]$. By correcting for the field produced by the oscillators on both sides of the surface he derived first-order expressions for $R_{\mathrm{p}}$ and $R_{\mathrm{s}}$. From these expressions the changes in $\Delta$ and $\psi$ due to the layer may be calculated. For uniaxially anisotropic layers $\left(\sigma_{x}=\sigma_{y} \neq \sigma_{z}\right)$ the results have been presented in ref. [6] [30]. For the general case $\sigma_{x} \neq \sigma_{y} \neq \sigma_{z}$, with $x z$ as plane of incidence, we get for $n_{0}=1$ and non-absorbing layers:

$$
\begin{aligned}
\delta \Delta & =8 \pi A\left\{\sigma_{z} B+\sigma_{x}\left[B\left(a^{2}-a^{\prime 2}-a / \sin ^{2} \phi_{0}\right)+a^{\prime 2} C^{2} / \sin ^{2} \phi_{0}\right]\right. \\
& \left.+\sigma_{y}\left[B\left(a \cot ^{2} \phi_{0}-a^{2}+a^{\prime 2}\right)+a^{\prime 2} C\left(2 a-\cot ^{2} \phi_{0}\right)\right]\right\} /\left(B^{2}+a^{\prime 2} C^{2}\right), \\
\delta \psi & =-4 \pi A \sin 2 \bar{\psi} a^{\prime}\left\{\sigma_{z} C-\sigma_{x}\left[B C / \sin ^{2} \phi_{0}+C\left(a / \sin ^{2} \phi_{0}-a^{2}+a^{\prime 2}\right)\right]\right. \\
& \left.-\sigma_{y}\left[B\left(2 a-\cot ^{2} \phi_{0}\right)+C\left(a^{2}-a^{\prime 2}-a \cot ^{2} \phi_{0}\right)\right]\right\} /\left(B^{2}+a^{\prime 2} C^{2}\right),
\end{aligned}
$$

with

$A=\frac{2 \pi}{\lambda} \cos \phi_{0} \sin ^{2} \phi_{0}, \quad B=\cos ^{2} \phi_{0}-a+\sin ^{2} \phi_{0}\left(a^{2}-a^{\prime 2}\right), \quad C=1-2 a \sin ^{2} \phi_{0}$, $a=\left(1-\kappa_{2}^{2}\right) / n_{2}^{2}\left(1+\kappa_{2}^{2}\right)^{2}, a^{\prime}=2 \kappa_{2} / n_{2}^{2}\left(1+\kappa_{2}^{2}\right)^{2}$.

As suggested before [6], for low oscillator strengths per unit surface area one can take

$$
\sigma_{j}=\alpha_{j} / c=\alpha_{j} \theta / c_{\mathrm{m}}, \quad j=x, y, z .
$$


For absorbing layers, i.e. if the scattering indices are complex $\left(\sigma_{j}=\sigma_{j}^{\prime}-\mathrm{i} \sigma_{j}^{\prime \prime}\right)$, eqs. (22) and (23) have to be extended in order to include terms with $\sigma_{x}^{\prime \prime}, \sigma_{y}^{\prime \prime}$ and $\sigma_{z}^{\prime \prime}$. In $\delta \Delta$ the coefficients of $\sigma_{j}^{\prime \prime}$ are equal to $(-2 / \sin 2 \bar{\psi})$ times the coefficients of $\sigma_{j}$ in eq. (23) and in $\delta \psi$ equal to $\frac{1}{2} \sin 2 \bar{\psi}$ times those of $\sigma_{j}$ in eq. (22). The coefficients of $\sigma_{j}^{\prime}$ are the same as those of $\sigma_{j}$ in eqs. $(22,23)$.

\subsection{Microscopic theories}

In the microscopic description given by Sivukhin $[12,13]$ a monomolecular transition layer at the surface is characterized by the parameters $\gamma_{j}$, defined as

$\gamma_{j}=\tau_{j} / P_{i}, \quad j=x, y, z$

where $\tau$ is the dipole moment induced in the layer per unit surface area, and $\boldsymbol{P}$ the polarization vector of the homogeneous substrate. For a review of the first-order expressions for $R_{\mathrm{p}}, R_{\mathrm{s}}, \delta \Delta$ and $\delta \psi$ in terms of the $\gamma_{j}$ 's for an uniaxially anisotropic layer $\left(\gamma_{x}=\gamma_{y} \neq \gamma_{z}\right)$ on an isotropic substrate see ref. [6]. Quentel and Kern [10] have extended the Sivukhin treatment to more general cases with several anisotropic layers on an anisotropic absorbing substrate.

The "surface susceptibility approach" presented by Dignam et al. $[31,32]$ for the calculation of the optical properties of thin planar arrays of molecules is similar but not identical to the treatment given by Sivukhin. Dignam et al. also characterized the layer at the surface by a parameter $\gamma$, defined differently, as a surface susceptibility tensor

$\gamma=d 4 \pi P / E^{0}$,

where $\boldsymbol{E}^{0}$ is the external field and $\boldsymbol{P}$ the mean polarization per unit volume in the layer with thickness $d$. The essential difference between the treatments of Sivukhin and Dignam et al. is that the latter consider the layer as a distinct phase with a finite thickness.

In these microscopic theories a central point is the calculation of the effective local field $E^{10 c}$ at a molecule in the transition layer. The effective field may be written as the sum of three terms

$E^{10 c}=E^{0}+E^{s}+E^{\prime}$.

The first term, $E^{0}$, is the homogeneous field (Sivukhin) or the external field (Dignam et al.) and is related to the macroscopic electric or Maxwell field, $\boldsymbol{E}$, through the boundary conditions

$E_{x, y}^{0}=E_{x, y}, \quad E_{z}^{0}=E_{z}+4 \pi P_{z}$.

The polarization contribution $P_{z}$ refers to the substrate in the description of Sivukhin and to the layer in the approach of Dignam. The second term in eq. (27), $E^{\mathrm{s}}$, represents the field of the dipoles in the transition layer

$E_{x, y}^{\mathrm{s}}=\left(A / 2 a^{3}\right) p_{x, y}, \quad E_{z}^{s}=-\left(A / a^{3}\right), p_{z}$ 
where $A$ is the two-dimensional lattice sum (e.g. $A=9.0336$ for a simple cubic lattice), $a$ the distance between the dipoles and $p$ the dipole moment of molecules in the layer $\left(p_{j}=\tau_{j} c\right)$. The third term in eq. (27), $\boldsymbol{E}^{\prime}$, is the "inhomogeneous field" of incident wave and substrate lattice (Sivukhin) or "interlayer local field contribution" (Dignam et al.). It has been estimated in different ways. According to Sivukhin, $\boldsymbol{E}^{\prime}$ is equal to half the difference between the field due to polarization alone in a thin slab of isotropic substrate material and the field of a two-dimensional dipole lattice of substrate atoms [12]. Quentel and Kern [10] calculated $\boldsymbol{E}^{\prime}$ as the field due to the substrate dipole layers. In the treatment of Dignam et al. the contribution of the substrate to the local field was approximated by considering dipole imaging in the substrate [32]. For nonmetals the contribution of $\boldsymbol{E}^{\prime}$ is mostly very small and negligible $[6,10]$. Dignam and Fedyk [32] have estimated that for metals the contribution of the images to the local field will usually also be small $(<10 \%)$.

With a reasoning analogous to that in section 2.3, Quentel and Kern [10] derived expressions for the dielectric constants of the adsorbed layers. For one uniaxially anisotropic layer on an isotropic substrate one gets

$\epsilon_{x, y}=n_{x, y}^{2}=1+4 \pi N \alpha_{x, y}\left(1+\Lambda_{x, y}\right) /\left(1-A \alpha_{x, y} / 2 a^{3}\right)$,

$\epsilon_{z}=n_{z}^{2}=1+4 \pi N \alpha_{z}\left(\epsilon_{2}-\Lambda_{z}\right) /\left(1+A \alpha_{z} / a^{3}\right)$.

Here the $\Lambda_{j}$ 's represent the correction terms due to the inhomogeneous field, containing the contributions from the substrate dipole layers. Neglecting $\Lambda_{j}$, these expressions reduce to those of Dignam (eq. (36)), if $\epsilon_{2}$ in eq. (31) is replaced by $\epsilon_{z}$. This difference arises from the inconsistent extrapolation of Sivukhin's approach to layers with a finite thickness.

The model of Dignam et al. basically expresses the dielectric tensor of a thin layer as

$\epsilon_{x, y}=\left(1+\alpha_{x, y}, \Sigma_{x, y}+4 \pi N \alpha_{x, y}\right) /\left(1+\alpha_{x, y} \Sigma_{x, y}\right)$.

$\epsilon_{z}=\left(1+\alpha_{z} \Sigma_{z}\right) /\left(1+\alpha_{z} \Sigma_{z}-4 \pi N \alpha_{z}\right)$,

where $N$ is again the number of molecules per unit volume and $\Sigma_{x, y}$ and $\Sigma_{z}$ are the dipole sums for dipoles parallel and perpendicular to the surface, which may include the effect of image dipoles. It is now useful to define a layer thickness $d$, which eliminates the parameter $\mathrm{N}$ in favour of $\mathrm{N}_{\mathrm{s}}(=\mathrm{Nd})$, the number of molecules per unit area, by demanding that $\epsilon_{x}=\epsilon_{z}$ for $\alpha_{x}=\alpha_{z}$. From eqs. (32) and (33) then follows

$d=\frac{4 \pi N_{s}}{\left(\Sigma_{z}-\Sigma_{x}\right)}$.

Neglecting the influence of image dipoles, the equations reduce to

$d=8 \pi N_{\mathrm{s}} / 3 t_{z}$, 
$\epsilon_{j}=1+\frac{4 \pi \alpha_{i} N_{\mathrm{s}} / d}{1-4 \pi / 3 \alpha_{j} N_{\mathrm{s}} / d}$

which is just the familiar Lorentz-Lorenz equation for a macroscopic phase (cf, eq. (18)). In eq. (35) $t_{z}$ is the lattice sum for real dipoles oriented perpendicular to the surface, equal to $A / a^{3}$ in eq. (29).

Including the effect of image dipoles leads to an effective thickness, given by

$d=8 \pi N / 3\left(t_{z}+\frac{1}{3} X v_{z}\right)$,

where $t_{z}$ is the direct dipole sum, $v_{z}$ the image dipole sum and $X$ the imaging parameter defined in terms of the substrate dielectric constant $\left(\epsilon_{2}\right)$ as

$X=\left(\epsilon_{2}-1\right) /\left(\epsilon_{2}+1\right)$

In this latter case no simple Lorentz-Lorenz type of equation is obtained.

The advantage of the approach of Dignam et al. is that it provides explicit expressions for the somewhat nebulous concept of the thickness of a monolayer. Moreover, as has been shown by Mahan and Lucas [33], the dipole sums for a randomly partially filled monolayer may be expressed as

$\Sigma(\theta)=\theta \Sigma(\theta=1)$,

which enables one to treat incomplete monolayers within this formalism. However, the inclusion of image dipoles may lead to complex values of the layer thickness (via the complex value of $X$ ). This follows from the assumption, that also in this case $\epsilon_{z}$ should equal $\epsilon_{x}$ if $\alpha_{z}=\alpha_{x}$, which is not physically obvious. Then this approach is difficult to reconcile with the use of the master equation, eq. (10).

As an example of the application of the different approaches we have calculated the ellipsometric effects of physical adsorption of oxygen molecules on the (100)

Table 1

Change in $\Delta$ calculated for adsorption of $\mathrm{O}_{2}$ molecules; angle of incidence $\phi_{0}=70^{\circ}$, wavelength $\lambda=6328 A_{3} \theta=\frac{1}{4} O_{2}$ molecule per surface metal atom; calculation for extrapolated maeroscopic model (EMM), Dignam's model (D) and Strachan's model (S)

\begin{tabular}{|c|c|c|c|c|c|c|}
\hline \multirow{2}{*}{ Substrate } & \multirow{2}{*}{$a(\AA)$} & \multirow{2}{*}{$\widetilde{n}_{2}$} & \multirow[t]{2}{*}{ Model } & \multicolumn{3}{|c|}{$\delta \Delta(\mathrm{deg})$} \\
\hline & & & & $\mathrm{O}_{2} \| z$ & $\mathrm{O}_{2} \| x$ & $\mathrm{O}_{2} \| y$ \\
\hline $\mathrm{Cu}$ & 5.11 & $0.13-\mathrm{i} 3.55[34]$ & $\begin{array}{l}\text { EMM } \\
D \\
S\end{array}$ & $\begin{array}{l}0.15 \\
0.17 \\
0.20\end{array}$ & $\begin{array}{l}0.10 \\
0.11 \\
0.12\end{array}$ & $\begin{array}{l}0.09 \\
0.10 \\
0.11\end{array}$ \\
\hline$A g$ & 5.78 & $0.054-i 4.54[35]$ & $\begin{array}{l}\text { EMM } \\
\text { D } \\
S\end{array}$ & $\begin{array}{l}0.14 \\
0.17 \\
0.19\end{array}$ & $\begin{array}{l}0.09 \\
0.10 \\
0.10\end{array}$ & $\begin{array}{l}0.09 \\
0.09 \\
0.10\end{array}$ \\
\hline $\mathrm{Ni}$ & 4.98 & $2.23-i 4.17[36]$ & $\begin{array}{l}\text { EMM } \\
\text { D } \\
S\end{array}$ & $\begin{array}{l}0.19 \\
0.23 \\
0.28\end{array}$ & $\begin{array}{l}0.12 \\
0.14 \\
0.15\end{array}$ & $\begin{array}{l}0.12 \\
0.13 \\
0.14\end{array}$ \\
\hline
\end{tabular}


surfaces of $\mathrm{Cu}, \mathrm{Ag}$ and $\mathrm{Ni}$. The polarizability of $\mathrm{O}_{2}$ was taken as $\alpha_{\|}=2.35 \AA^{3}$ and $\alpha_{\perp}=1.21 \AA^{3}$ and the dimensions as $l=2.54 \AA$ and $D=1.32 \AA$. The coverage is $\frac{1}{4}$ $\mathrm{O}_{2}$ per surface metal atom in a $(2 \times 2)$ arrangement with $A=9.0336$ and $c=a^{2}$, where $a$ is $\sqrt{ } 2$ times the lattice constant of the metals. The calculations were carried out with the extrapolated macroscopic model (EMM, eqs. (18) and (21)), Strachan's model (S, eqs. (22)-(24)) and Dignam's model (D, eqs. (35) and (36)). The calculated $\delta \psi$ values were always negative and larger than -0.03 . The $\delta \Delta$ values are given in table 1. As calculated before for isotropic layers [6], Strachan's model yields the highest values.

All models show a significantly higher $\delta \Delta$ for molecules adsorbed with their long axis perpendicular to the surface. Molecules with $l$ parallel to the surface show a slightly higher $\delta \Delta(5-10 \%)$ if the length axis lies in the plane of incidence.

\section{Clean surfaces}

As a result of the tendency of minimalization of the Gibbs energy, the geometric and electronic structure of the surface region of a crystal is often different from the bulk structure. This has not only been established for substances with strong covalent bonds (superstructures and surface states of semiconductors such as silicon and germanium [37]) but also for metal surfaces. Studies with surface-sensitive techniques such as LEED and MEIS (medium energy ion scattering) have shown that most clean metal surfaces are in lateral registry with the truncated bulk but exhibit a tendency towards small bond length contraction between the first and next atomic layers, especially on the less close-packed faces [38] E.g., for $\mathrm{Ag}(110)$ the contraction of the first layer spacing compared to the bulk value has been found to be $8 \%$ [39], for $\mathrm{Cu}(110) 10 \%$ [40] and for $\mathrm{Ni}(110) 5 \%$ [41,42], whereas for the closer-packed (111) and (100) planes of the same metals within experimental accuracy $( \pm 3 \%)$ no contraction has been established. In general adsorption causes relaxation of the top substrate layer back to the bulk arrangement or even further outwards [38].

In principle the optical properties $(\tilde{n}, \epsilon)$ of a restructured surface region will also differ from those of the homogeneous bulk. The ellipsometric results obtained on chemisorption of various gases on clean semiconductor surfaces could be described with a model in which a thin transition layer at the clean surface, with optical properties associated with surface states, is effectively removed [6-8].

For clean metal surfaces with different extent of contraction or expansion between the outer atomic layers one would expect to find different values of the ellipsometric parameters $\bar{\Delta}$ and $\bar{\psi}$. The results of a simplified calculation of these differences for the examples mentioned above are shown in table 2 . Here we have assumed that the (110) surface is identical to (111) with on top a layer with thickness equal to the contracted distance, $d_{2}^{\mathrm{ss}}=p \cdot d_{(110)}$, between successive (110) planes and index of refraction $\widetilde{n}_{2}^{\text {ss }}$, related to $\widetilde{n}_{2}$ by the Maxwell Garnett equation 
Table 2

Calculated differences in ellipsometric parameters between (110) and (111) surfaces; $\phi_{0}, \lambda$ and $\widetilde{n}_{2}$ as in table 1

\begin{tabular}{llllll}
\hline & $p$ & $\begin{array}{l}d_{2}^{\text {ss }} \\
(\AA)\end{array}$ & $\widetilde{n}_{2}^{\text {ss }}$ & $\begin{array}{l}\bar{\Delta}_{(110)}-\bar{\Delta}_{(111)} \\
(\mathrm{deg})\end{array}$ & $\begin{array}{l}\bar{\psi}_{(110)}-\bar{\psi}_{(111)} \\
(\mathrm{deg})\end{array}$ \\
\hline $\mathrm{Ag}$ & 0.92 & 1.33 & $0.19-\mathrm{i} 6.77$ & 0.34 & -0.01 \\
$\mathrm{Cu}$ & 0.90 & 1.15 & $0.30-\mathrm{i} 4.61$ & 0.15 & -0.01 \\
$\mathrm{Ni}$ & 0.95 & 1.18 & $3.26-\mathrm{i} 3.90$ & -0.01 & -0.06 \\
\hline
\end{tabular}

[43]

$\left(\tilde{n}_{2}^{s s^{2}}-1\right) /\left(\widetilde{n}_{2}^{s s^{2}}+2\right)=p^{-1}\left(\widetilde{n}_{2}^{2}-1\right) /\left(\widetilde{n}_{2}^{2}+2\right)$.

By probing with electrons or ions the surface structure can be established in single experiments with clean surfaces. However, the larger penetration depth of light and special experimental problems (e.g. window errors and manipulator stability) enable only accurate relative optical measurements, i.e. a combination of two successive measurements (electromodulation, adsorption). It will take very special experimental efforts to establish differences in absolute values of $\Delta$ and $\Psi$ of the order of magnitude as given in table 2 between two samples with confidence. Corrections for window birefringence may be determined from measurements with clearl and contaminated surfaces in vacuum and at $1 \mathrm{~atm}$ air and with contaminated surfaces at 1 atm with and without cell windows $[35,44]$. However, irreproducibilities in birefringence and oxidation of the surface, and mechanical instabilities of the vacuum setup and manipulator may invalidate this procedure. For the metals of table 1 a change in the angle of incidence $\phi_{0}\left(\approx 70^{\circ}\right)$ with $\pm 0.5^{\circ}$ will cause a change in $\bar{\Delta}$ of $\overline{+} 1.5^{\circ}$ and in $\bar{\psi}$ of $+0.01^{\circ}$ for $\mathrm{Cu}$ and $\mathrm{Ag}$ and $\overline{+} 0.4^{\circ}$ for $\mathrm{Ni}$.

In view of these potential experimental errors it is not astonishing that values of $\widetilde{n}$ for clean surfaces vary considerably in the literature. Other sources of variation are differences in the physical state of the samples (film, single crystal) and the topography of the surface [45], and in cleanliness of surface and bulk. The low sensitivity of ellipsometry for adsorbed layers $-10^{-1}-10^{-2} \mathrm{ML}$ (monolayers), as compared to $10^{-2}-10^{-4} \mathrm{ML}$ for electron spectroscopy, $10^{-3}-10^{-6} \mathrm{ML}$ for ion scattering and secondary ion mass spectroscopy - combined with low elemental specificity make this method in general less suitable for monitoring surface contamination. Optical studies have to be combined with other surface analytical techniques to establish surface cleanliness.

As an example experimental values determined for $\mathrm{Ag}$ and $\mathrm{Cu}$ at $\lambda=6328 \AA$ are shown in fig. 2. Unannealed films, polycrystalline samples and ion-bombarded single crystal surfaces show a tendency to larger $n$ and smaller $k$ valucs. Albers et al. [35] found that argon ion bombardment of $\mathrm{Ag}(111)$ caused a decrease in $\Delta$ and $\psi$, which could be interpreted as an increase in $n$ and decrease in $k$ of the damaged sur- 


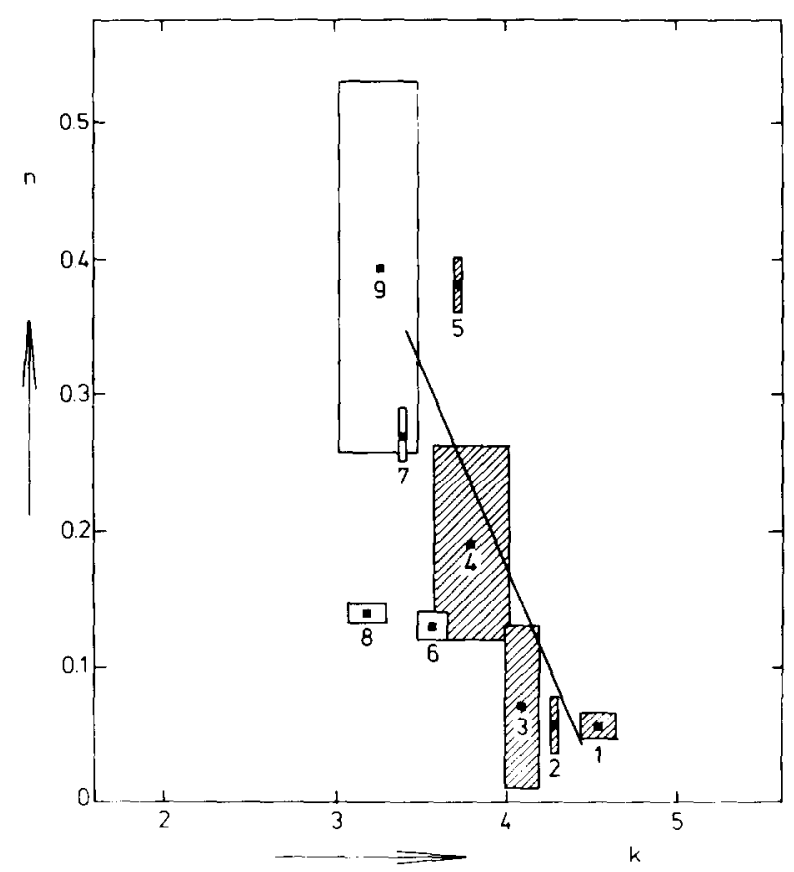

Fig. 2. Optical constants of $\mathrm{Cu}$ (open rectangles) and $\mathrm{Ag}$ (shaded rectangles) at $\lambda=6328 \AA$ : (1) single crystal in UHV [35], (2) film in UHV [46], (3) single crystal in electrolyte [47], (4) polycrystalline in electrolyte [48], (5) polycrystalline in electrolyte [49], (6) single crystal in UHV [34], (7) film in UHV [46], (8) polycrystalline in UHV [50], (9) polycrystalline in electrolyte [47]. The drawn line is for ion-bombarded Ag [35].

face region. The optical constants calculated for the damaged layer at the surface correspond to those of unannealed thin films.

\section{Submonolayer and monolayer films}

\subsection{Chemisorption on metal surfaces}

Ellipsometric studies of (sub)monolayer chemisorption on metal surfaces are scarce. Chou et al. [51] investigated the initial growth of a surface layer on a clean lead film exposed to oxygen. Their data could be explained with a constant refractive index of the film, even down to effective thicknesses of $0.4 \AA$. The refractive index agreed remarkably well with the bulk refractive index of $\mathrm{PbO}$. Results obtained by Carroll and Melmed for oxygen adsorption on W(011) [52] and on $\mathrm{Fe}(011)$ [53] pointed to more complicated explanations involving geometric and 
electronic changes in the substrate. Such a conclusion has also been reached from an ellipsometric study of the adsorption of $\mathrm{CO}$ on nickel [54] and copper [55] films. From an ellipsometric study at different wavelengths of the adsorption of oxygen on evaporated silver films O'Handley and Burge [56] inferred the existence of adsorbate-induced surface states.

Rubloff et al. have performed optical reflectance spectroscopic investigations of the chemisorption of $\mathrm{O}_{2}, \mathrm{CO}$ and $\mathrm{H}_{2}$ on $\mathrm{Mo}(100)$ [57] and $\mathrm{H}_{2}$ on $\mathrm{W}(100)$ [58]. The structure that appeared in the reflectance change as a function of coverage was attributed to different binding configurations of the ad-atoms, as also observed with LEED, and the structure in the wavelength dependence $(1.9-4.8 \mathrm{eV})$ of the reflectance change was suggested to originate from adsorbate-induced surface states. Watanabe and Hirakuta [59] have calculated the effect of chemisorption on the optical reflection and electrical resistance of thin $\mathrm{Ag}$ films by estimating the scattering probabilities of the conduction electrons by chemisorbed atoms with quantum mechanical scattering theory. A model to take surface roughness into account has been developed by Dignam et al. $[60,61]$ and used to analyze the transmission and reflectance spectra of gases adsorbed on silver films. With this model and by allowance for the direction of polarization of absorption bands in polarization modulation spectra, Moskovits and McBreen were able to deduce the surface geometry of hydrogen and ethylene adsorbed on copper films [62].

Recently Wyrobisch has studied the initial oxidation of aluminum single crystal surfaces with ellipsometry and other surface techniques [63]. The changes in $\Delta$ were found to correlate with changes in the oxygen Auger signal and to yield sensitive information about the amount of adsorbed and/or incorporated oxygen. The large positive values of $\delta \psi\left(0.1-0.2^{\circ}\right)$ could not be explained by applying the extrapolated macroscopic theory or the approaches by Strachan and Sivukhin as formulated in ref. [6].

With the combination ellipsometry-AES-LEED linear relationships have been observed between the change in $\Delta$ at $\lambda=632.8 \AA$ and the oxygen coverage $\theta$ in the chemisorption stage $(\theta \leqslant 0.5$ oxygen atoms/metal surface atom) on $\mathrm{Ag}(110)$ [64], $\mathrm{Cu}(111)$ [65], $\mathrm{Cu}(110)$ [66] and $\mathrm{Cu}(100)$ [67]. This has been applied to determine the kinetics of the adsorption of $\mathrm{O}_{2}$ and $\mathrm{N}_{2} \mathrm{O}$ and of the removal of adsorbed oxygen by $\mathrm{CO}$. For $\mathrm{Ni}(100)$ the ellipsometric changes in the first, chemisorption stage were too small to derive a reliable coverage dependence [68]. After the first stage a further, generally much slower uptake (incorporation) of oxygen takes place on $\mathrm{Cu}$ $[67,69]$ and $\mathrm{Ni}[68]$, accompanied by reconstruction of the surface. The ellipsometric effects per incorporated atom are smaller for $\mathrm{Cu}$ and larger for Ni. For copper at coverages $0.5<\theta<2$ the change in $\psi$ is zero within experimental error $\left(\leqslant 0.02^{\circ}\right)$.

A summary of observed changes in $\Delta$ and $\psi$ is given in table 3 . At room temperature the $\delta \Delta$ values per oxygen atom adsorbed on the different $\mathrm{Cu}$ planes are equal within the experimental accuracy (10\%). For the anisotropic $\mathrm{Cu}(110)$ plane the $\delta \Delta$ and $\delta \psi$ values per adsorbed oxygen atom depend on the crystal temperature 
Table 3

Ellipsometric effects measured upon adsorption of oxygen at room temperature, $\lambda=6328 \AA$

\begin{tabular}{|c|c|c|c|c|c|c|c|c|}
\hline \multirow[t]{2}{*}{ Substrate } & \multirow{2}{*}{$\begin{array}{l}\phi_{0} \\
\text { (deg) }\end{array}$} & \multirow{2}{*}{$\begin{array}{l}s z \\
\text { (deg) }\end{array}$} & \multicolumn{2}{|c|}{$\theta=0.5^{\mathrm{a}}$} & \multirow{2}{*}{$\begin{array}{l}\theta=1.0 \\
\delta \Delta \\
(\mathrm{deg})\end{array}$} & \multirow{2}{*}{$\begin{array}{l}\theta=1.5 \\
\delta \Delta \\
(\mathrm{deg})\end{array}$} & \multicolumn{2}{|c|}{$\theta=2.0$} \\
\hline & & & $\begin{array}{l}\delta \Delta \\
\text { (deg) }\end{array}$ & $\begin{array}{l}\delta \psi \\
\text { (deg) }\end{array}$ & & & $\begin{array}{l}\delta \Delta \\
(\mathrm{deg})\end{array}$ & $\begin{array}{l}\delta \psi \\
\text { (deg) }\end{array}$ \\
\hline $\mathrm{Ag}(110)$ & 71 & $\sim 45$ & 0.45 & 0.12 & & & & \\
\hline $\mathrm{Cu}(111)$ & 69 & & 0.68 & 0.22 & 0.98 & & & \\
\hline $\operatorname{Cu}(100)$ & 69 & & 0.65 & 0.30 & 0.80 & & & \\
\hline \multirow[t]{2}{*}{$\mathrm{Cu}(110)$} & 69 & 0 & 0.49 & 0.30 & 0.70 & 0.92 & 1.13 & 0.30 \\
\hline & 69 & 90 & 0.49 & 0.12 & 0.70 & & & \\
\hline $\mathrm{Ni}(100)$ & 68 & & 0.04 & 0.00 & & & 1.65 & 0.09 \\
\hline
\end{tabular}

${ }^{\mathrm{a}}$ Except for $\mathrm{Cu}(111)$, where $\theta=0.45$.

and the azimuth $\Omega$, defined as the angle between the plane of incidence of the light beam and the rows on the surface ([ $\overline{1} 10]$ direction). This anisotropy will be discussed in section 4.3 . In order to explain the observed changes in $\Delta$ and $\psi$, notably in the first stage, in the next section optical models will be examined in which changes in the substrate, caused by the chemisorption of oxygen, are taken into account.

\subsection{Models for chemisorption of oxygen on metal surfaces}

The optical models which will be considered are schematically shown in fig. 3 .

Model $a$. In the model used to interpret the ellipsometric results obtained for chemisorption of gases on semiconductor surfaces [6-8] it was assumed that the measured effects consist of a contribution from the adsorbed layer itself and a contribution from changes in the substrate surface region (fig. 3a):

$\delta \Delta_{\text {obs }}=\delta \Delta_{\mathrm{ad}}+\delta \Delta_{s s}$,

$\delta \psi_{\mathrm{obs}}=\delta \psi_{\mathrm{ad}}+\delta \psi_{\mathrm{ss}}$.

The contribution from the adsorbed layer was calculated in the same way as for physisorbed, non-absorbing layers, i.e. with eq. (20). The effective optical constants of the surface-states layer, $n_{2}^{\text {ss }}$ and $k_{2}^{\text {ss }}$, were derived from $\delta \Delta_{\mathrm{ss}}$ and $\delta \psi_{\mathrm{ss}}$, by choosing a value for the thickness $d_{\mathrm{ss}}$ of this layer. For semiconductors this modcl yielded reasonable results, comparable to those obtained by other methods [7].

Table 4 presents results of calculations for chemisorption of oxygen atoms and ions on some metal surfaces. Using the computer program of McCrackin [70] for $\mathrm{Cu}$ and $\mathrm{Ag}$ no physically reasonable values for the optical constants $n_{2}^{\text {ss }}$ and $k_{2}^{\text {ss }}$ could be obtained $\left(d_{\mathrm{ss}}=2-5 \AA\right)$. Solutions obtained for $\mathrm{Ni}$ are e.g. $\tilde{n}_{2}^{\mathrm{ss}}=2.1-\mathrm{i} 3.9$ 


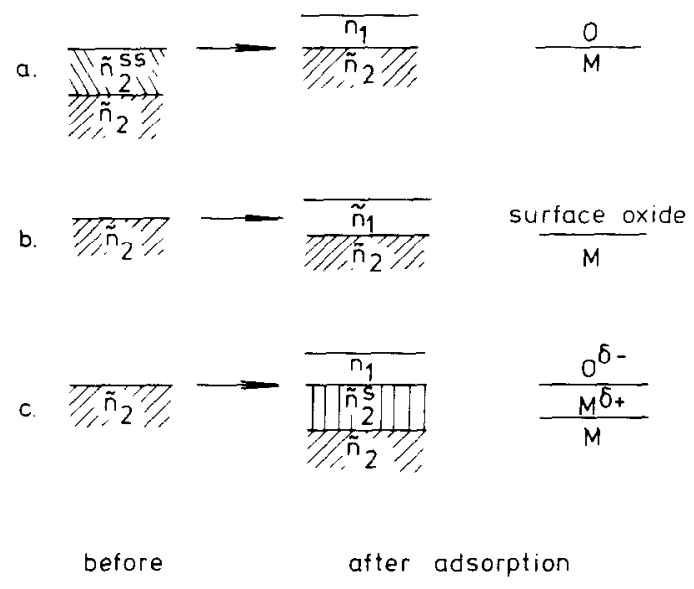

Fig. 3. Optical models for chemisorption.

for atoms and $1.6-i 1.9$ for ions, both with $d_{\mathrm{ss}}=4 \AA$ ( $\sim 2$ atomic layers). Comparison of the values for $\delta \Delta_{\mathrm{ss}}$ and $\delta \psi_{\mathrm{ss}}$ from table 4 with those calculated for surface relaxation (cf. table 2) shows that such a version of model a cannot explain the data: the sign as well as the order of magnitude of $\delta \psi$ are wrong. Moreover one would expect that this effect is plane-specific, whereas the data obtained for $\mathrm{Cu}$ only show plane-specificity in $\delta \psi$.

Model $b$. The simplest interpretation of the experimental data is in principle the one-layer model (fig. 3b): formation of a surface oxide with effective index of refraction $\widetilde{n}_{1}$ and thickness $d_{1}$. The values calculated for $n_{1}$ and $k_{1}$ depend of course on the assumed thickness of the layer. At the end of the chemisorption stage $\left(\theta \approx \frac{1}{2}\right), d_{1}$ will be of the order of the distance between two atomic layers in the metal or oxide $(\sim 2 \AA)$. For the experimentally observed $\Delta, \psi$ values (table 3 ) two solutions are obtained with McCrackin's program [70], of which fig. 4 gives an

Table 4

Calculations with model a (fig. $3 \mathrm{a})$ for adsorption of oxygen atoms $\left(\alpha=0.79 \AA^{3}, d_{\mathrm{m}}=1.32 \AA\right.$ ) and ions $\left(\alpha=3.88 \AA^{3}, d_{\mathrm{m}}=2.92 \AA\right)$ with data of table 3

\begin{tabular}{llllll}
\hline Substrate & $\begin{array}{l}c_{\mathrm{m}} \\
\left(\AA^{2}\right)\end{array}$ & $\theta$ & $\begin{array}{l}\delta \Delta_{\mathrm{ss}}(\mathrm{deg}) \\
\mathrm{O}\end{array}$ & $\begin{array}{l}\delta \Delta_{\mathrm{ss}}(\mathrm{deg}) \\
\mathrm{O}^{2-}\end{array}$ & $\begin{array}{l}\delta \psi \text { ss (deg) } \\
\mathrm{O}, \mathrm{O}^{2-}\end{array}$ \\
\hline $\mathrm{Ag}(110)$ & 11.83 & 0.5 & 0.37 & 0.13 & 0.12 \\
$\mathrm{Cu}(111)$ & 5.64 & 0.45 & 0.57 & 0.25 & 0.22 \\
$\mathrm{Cu}(100)$ & 6.52 & 0.5 & 0.55 & 0.23 & 0.30 \\
$\mathrm{Ni}(100)$ & 6.20 & 0.5 & -0.09 & -0.45 & $\sim 0.02$ \\
\hline
\end{tabular}




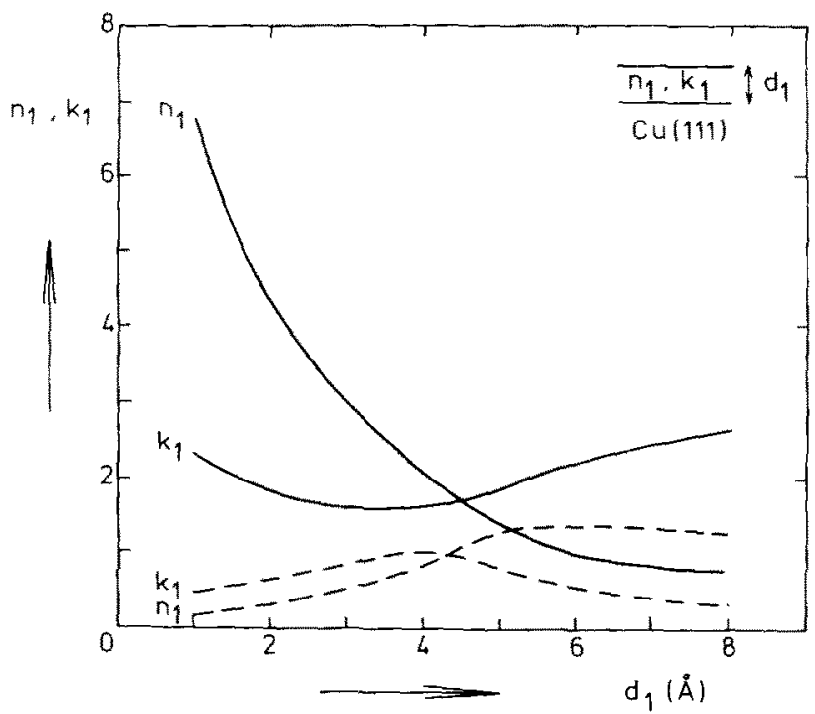

Fig. 4. Thickness dependence of $n_{1}$ and $k_{1}$ for oxygen on Cu(111) (table $3_{*} \theta=0.45$ ). Two different solutions are denoted by solid and dashed lines.

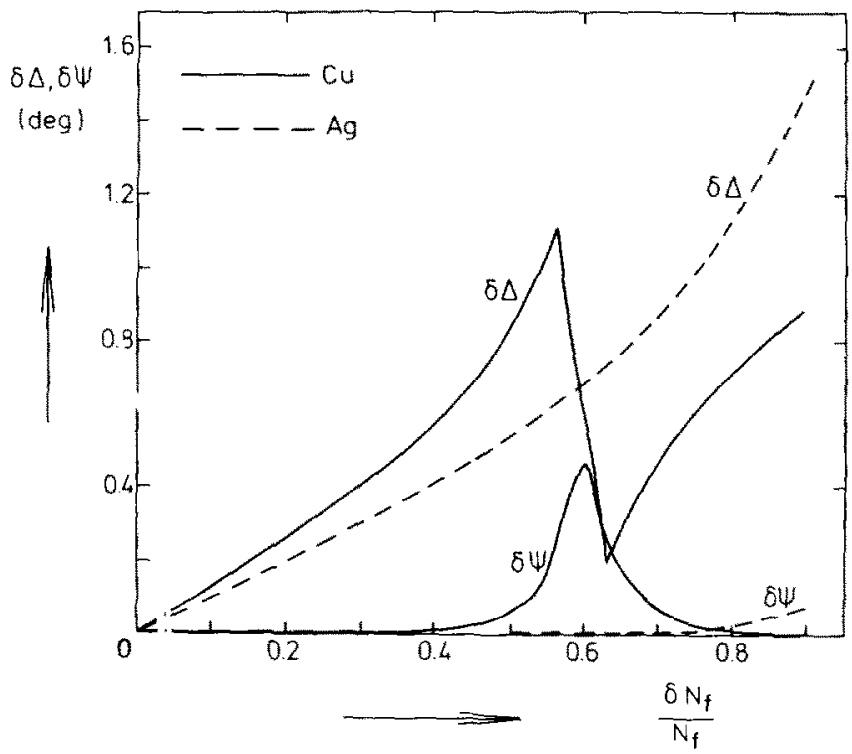

Fig. 5. $\delta \Delta$ and $\delta \psi$ for $\mathrm{Cu}(--\rightarrow$ and $\mathrm{Ag}(---)$ as a function of the relative decrease in free electron density in a surface laye if $4.2 \AA ; \lambda=6328 \AA, \phi_{0}=70^{\circ}$. 
Table 5

Values of $n_{1}$ and $k_{1}$ calculated with model b (fig. $3 \mathrm{~b}$ ) for the experimental data of table 3 ; thicknesses were arbitrarily chosen as $4 \AA /$ monolayer

\begin{tabular}{lcccccc}
\hline$\theta$ & $d_{1}(\AA)$ & $\operatorname{Ag}(110)$ & $\mathrm{Cu}(111)$ & $\mathrm{Cu}(100)$ & $\mathrm{Cu}(110), \Omega=0^{\circ}$ & $\mathrm{Ni}(100)$ \\
\hline 0.5 & 2 & $2.7-\mathrm{i} 2.0$ & $4.3-\mathrm{i} 1.8$ & $4.5-\mathrm{i} 2.4$ & $4.0-\mathrm{i} 2.8$ & $2.1-\mathrm{i} 4.0$ \\
1.0 & 4 & & $3.1-\mathrm{i} 1.2$ & $2.8-\mathrm{i} 1.9$ & $2.5-\mathrm{i} 2.0$ & \\
1.5 & 6 & & & & $1.8-\mathrm{i} 1.6$ & \\
2.0 & 8 & & & & $1.5-\mathrm{i} 0.8$ & $2.6-\mathrm{i} 0.5$ \\
\hline
\end{tabular}

example. Table 5 presents the most acceptable set of $n_{1}, k_{1}$ combinations for various coverages.

For thicker films $\left(d_{1}>100 \AA\right)$ of $\mathrm{Cu}_{2} \mathrm{O}$ on copper values have been reported of $\tilde{n}_{1}=(2.3-3.0)-\mathrm{i}(0.1-0.3)$ at $\lambda=5461 \AA[71,72]$, at $\lambda=6328 \AA$ probably $k \simeq 0$ [73]; for bulk NiO $\widetilde{n}=2.33-\mathrm{i} 0.00$ at $\lambda=6328 \AA$ [74]. The values calculated for $\theta=\frac{1}{2}$ are distinctly different; $k$ especially is much higher. If we assume that after the chemisorption stage an oxide layer is formed on top of the copper substrate with effective $\tilde{n}_{2}$, calculated from $\Delta$ and $\psi$ at $\theta=\frac{1}{2}$, we get for $\tilde{n}_{1}: 2.2-$ i0.03.

In order to rationalize the data at $\theta=\frac{1}{2}$ we have performed Maxwell Garnett calculations for the metal-oxygen system, using

$\frac{\epsilon_{1}-1}{\epsilon_{1}+2}=\frac{4}{3} \pi N_{\mathrm{M}} \alpha_{\mathrm{M}}+\frac{4}{3} \pi N_{0} \alpha_{0}$,

where $\epsilon_{1}$ is the dielectric constant of the metal oxide, $N_{\mathrm{M}}$ and $N_{0}$ the number density of metal atoms and oxygen atoms and $\alpha_{M}$ and $\alpha_{0}$ their polarizabilities. As regards the oxides, three different assumptions have been tried viz. (i) the oxygen atoms simply replace metal atoms at constant total number density, (ii) oxygen atoms are inserted interstitially in the metal lattice at constant total density, and (iii) the density of the mixed system is allowed to vary linearly between that of the pure metal and that of the pure metal oxide. For none of the metals studied agreement could be obtained between "observed" and calculated optical constants, allowing for a variable amount of oxygen and "observed" layer thicknesses of 1-8 $\AA$, with the three assumptions outlined above. A similar conclusion has been drawn by Wyrobisch for the $\mathrm{Al} / \mathrm{O}$ system [63]. It thus seems that, contrary to the case of $\mathrm{Pb} / \mathrm{O}$ [51], for these metals no simple interpretation of the optical properties can be given, at least for the chemisorption stage.

With Strachan's model (section 2.4) the observed $\delta \psi$ values can only be explained with complex scattering indices. Using eqs. (22)-(24) for absorbing, isotropic layers $\left(\sigma_{x}=\sigma_{y}=\sigma_{z}\right)$ one gets from the data of table 3 at $\theta=\frac{1}{2}$, e.g. for $\mathrm{Cu}(111): \alpha=3.6-\mathrm{i} 2.5, \mathrm{Cu}(110): \alpha=3.7-\mathrm{i} 3.5$, and $\mathrm{Ag}(110): \alpha=3.8-\mathrm{i} 2.1$.

Model $c$. The two-layer model (fig. 3c) with adsorbed oxygen ions on top of an 
effectively positively charged metal surface layer can be considered as a detailed version of model $\mathrm{b}$. The effective refractive index $n_{1}$ is estimated as in model a with eq. (20); the polarizability of the adsorbed species will depend on the ionic charge $\delta$.

The optical constants $n_{2}^{\mathrm{s}}$ and $k_{2}^{8}$ of the topmost metal layer may differ from the bulk values due to a decrease in the free electron density $N_{\mathrm{f}}$, and/or to a decrease in the collision time $\tau$, if we assume that the contribution of the bound electrons remains constant upon chemisorption. Apart from the changes in $\tau$ this description is the same as in the McIntyre-Aspnes model, proposed for electroreflectance at metal surfaces [75]. Changes in $\tau$ have also been considered by Dignam and Moskovits [60], to account for surface roughness.

Using the formulae and optical data in the Appendix we have calculated the optical constants $n_{2}^{\mathrm{s}}$ and $k_{2}^{\mathrm{s}}$ of a thin layer as a function of $N_{\mathrm{f}}$ and $\tau$, and subsequently the changes in $\Delta$ and $\psi$. Fig. 5 shows how $\delta \Delta$ and $\delta \psi$ depend on the fraction of removed free electrons $\left(\delta N_{\mathrm{f}} / N_{\mathrm{f}}\right)$, for an arbitrarily chosen thickness of $4.2 \AA\left(\sim 2\right.$ atomic layers). For copper $\delta \Delta$ increases up to $\delta N_{\mathrm{f}} / N_{\mathrm{f}}=0.55$, while $\delta \psi$ remains very small $\left(\leqslant 0.01^{\circ}\right)$ up to $\delta N_{\mathrm{f}} / N_{\mathrm{f}}=0.4$. The structure in the curves around $\delta N_{\mathrm{f}} / N_{\mathrm{f}} \simeq 0.6$ corresponds to $\epsilon^{\prime}=0$. For silver qualitatively the same curves are found, except that $\epsilon^{\prime}=0$ in the region where $90-100 \%$ of the free electrons are removed.

Fig. 6 shows how the ellipsometric changes depend on the collision time $\tau$ at constant electron densities $\left(\delta N_{\mathrm{f}} / N_{\mathrm{f}}=0\right.$ and 0.4$)$. Down to $\tau \simeq 4 \times 10^{-15} \mathrm{~s}, \Delta$ and $\psi$

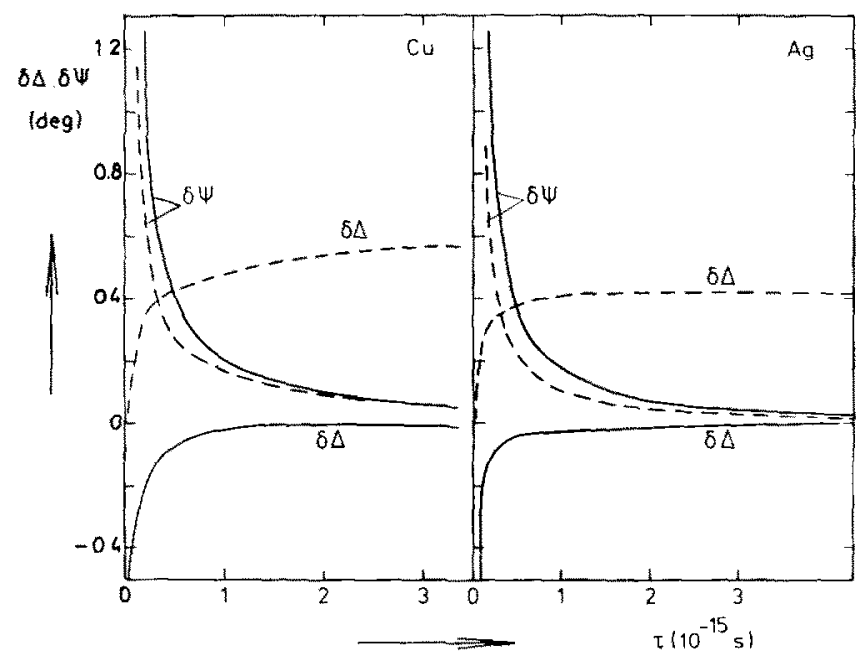

Fig. 6. $\delta \Delta$ and $\delta \psi$ for $\mathrm{Cu}$ (left) and $\mathrm{Ag}$ (right) as a function of the collision time $\tau ;(\longrightarrow)$ $\delta N_{\mathrm{f}} / N_{\mathrm{f}}=0,(--) \delta N_{\mathrm{f}} / N_{\mathrm{f}}=0.4$. 
remain constant and for smaller $\tau, \delta \psi$ increases and $\delta \Delta$ decreases. For $\tau>10^{-15} \mathrm{~s}$, the changes in $\Delta$ due to changes in $N_{\mathrm{f}}$ and $\tau$ appear to be additive.

The total changes calculated with this model may in good approximation be taken as

$\delta \Delta_{\mathrm{calc}}=\delta \Delta_{0}+\delta \Delta_{N_{\mathrm{f}}}+\delta \Delta_{\tau}$,

$\delta \psi_{\mathrm{calc}}=\delta \psi_{0}+\delta \psi_{N_{\mathrm{f}}}+\delta \psi_{\tau}$.

The three terms denote the changes due to the layer of oxygen ions, and the decrease in density and collision time of the free electrons. The contribution $\delta \Delta_{0}$ depends on the ionic charge and will be positive; $\delta \psi_{0}$ is negative, but negligibly small (cf. table 4). A fit with the experimental data (table $3, \theta=0.5$ ) may be obtained by ascribing $\delta \psi$ totally to the reduction in $\tau$ (or mean free path) and $\delta \Delta$ to the charge transfer $\left(\delta \Delta=\delta \Delta_{0}+\delta \Delta_{N_{\mathrm{f}}}\right)$; e.g. for $\operatorname{Ag}(110)$ and $\mathrm{Cu}(111)$ one gets the observed values with $\tau \approx 0.7-1.4 \times 10^{-15} \mathrm{~s}, \delta N_{\mathrm{f}} / N_{\mathrm{f}} \approx 0.1$ (or $\delta \approx 0.4$ ) and $\alpha \approx$ $5 \AA^{3}$, for a layer thickness of $4.2 \AA$. These values are not unreasonable and do indeed reproduce the experimental data, although not too much significance may be attached to their precise magnitudes.

In fig. 7 the experimental $\delta \psi$ is plotted against $\delta \Delta$ (proportional to the oxygen coverage) for $\mathrm{Cu}(111), \mathrm{Cu}(100)$ and for $\mathrm{Cu}(110)$ at different azimuth; $\delta \psi$ increases with $\delta \Delta$, but on $\mathrm{Cu}(100)$ and on $\mathrm{Cu}(110)$ at $\Omega=0^{\circ}$ becomes constant at $\delta \psi=0.3^{\circ}$. Within the framework of model $\mathrm{c}$ this saturation effect can be ascribed to constructive interference of the scattered electrons at a distance between the scattering centres equal to or smaller than the wavelength of the conduction clectrons 176 ,

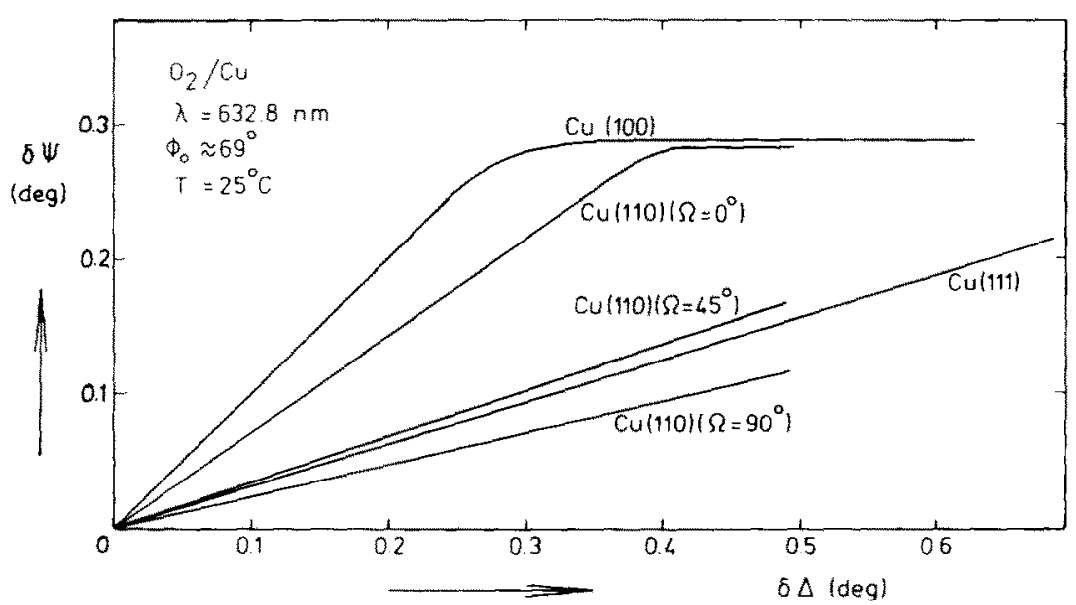

Fig. 7. Experimental $\delta \psi$ versus $\delta \Delta$ for chemisorption of oxygen on various copper planes. The endpoints of the curves represent the completion of the chemisorption stage. 
59]. In principle fig. 7 then contains information about the spatial distribution of the ad-atoms at different coverages, comparable with that resulting from resistance measurements.

\subsection{Anisotropy}

In principle one expects even a clean surface to exhibit anisotropy if the site symmetry contains no fourfold or higher order symmetry axis. This should manifest itself in different $\bar{\Delta}$ and/or $\bar{\psi}$ values if the crystal is rotated along an axis normal to the surface. This effect may be difficult to measure due to experimental difficulties (section 3). However, electroreflectance measurements on $\mathrm{Ag}(110)$ [77] and $\mathrm{Cu}(110)$ [78] in electrolyte solution have revealed optical anisotropy, which was explained in terms of direction-dependent surface conductivities [78].

A different type of anisotropy is the observation that $\delta \Delta$ and/or $\delta \psi$ values depend on the direction of the incident plane of the light beam. This effect has been observed with thick oxide films (100-1200 $\AA$ ) on copper single crystals with (110) and (311) orientations [79,71] and may be associated with anisotropic strain in the plane of the oxide film [80]. With differential reflectance spectroscopy Kolb et al. [81] observed for deposition of a (sub)monolayer of $\mathrm{Cu}$ on $\mathrm{Pt}(110)$ a pronounced anisotropy when the electric field vector of a linearly polarized light beam was rotated. A similar, ellipsometric effect has been observed in our work with submonolayer coverages of oxygen on $\mathrm{Cu}(110)$ (table 3 , fig. 7). In fig. $8, \delta \Delta$ and $\delta \psi$, both at $\theta=0.5$, are shown for different values of the azimuth $\Omega$ of the plane of incidence and different crystal temperatures. Fig. 9 shows $\delta \Delta$ as a function

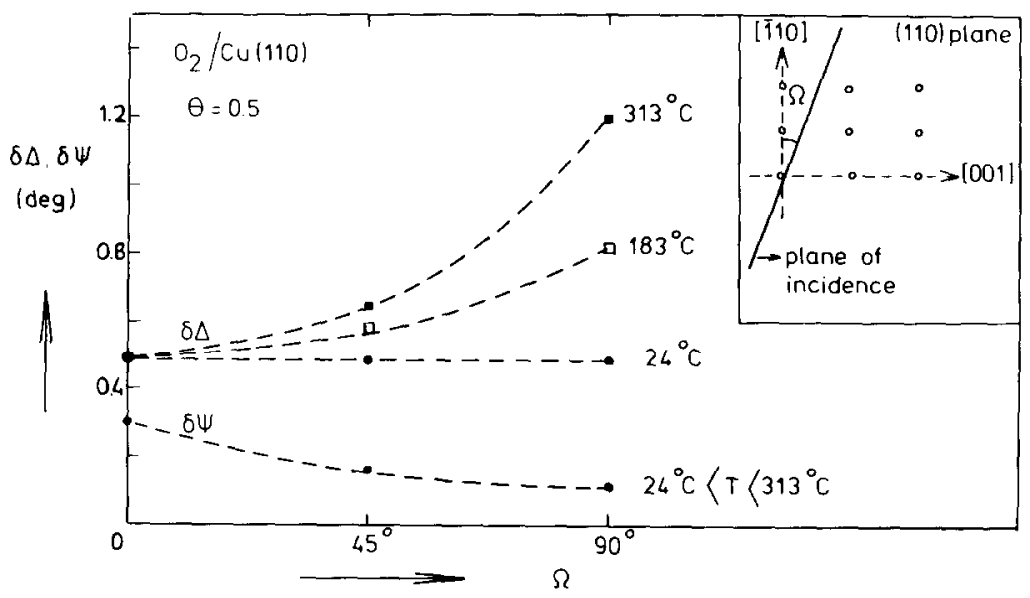

Fig. 8. Experimental $\delta \Delta$ and $\delta \psi$ of half a monolayer coverage of oxygen on $\mathrm{Cu}(110)$ as a function of the azimuth $\Omega$ at different temperatures. 


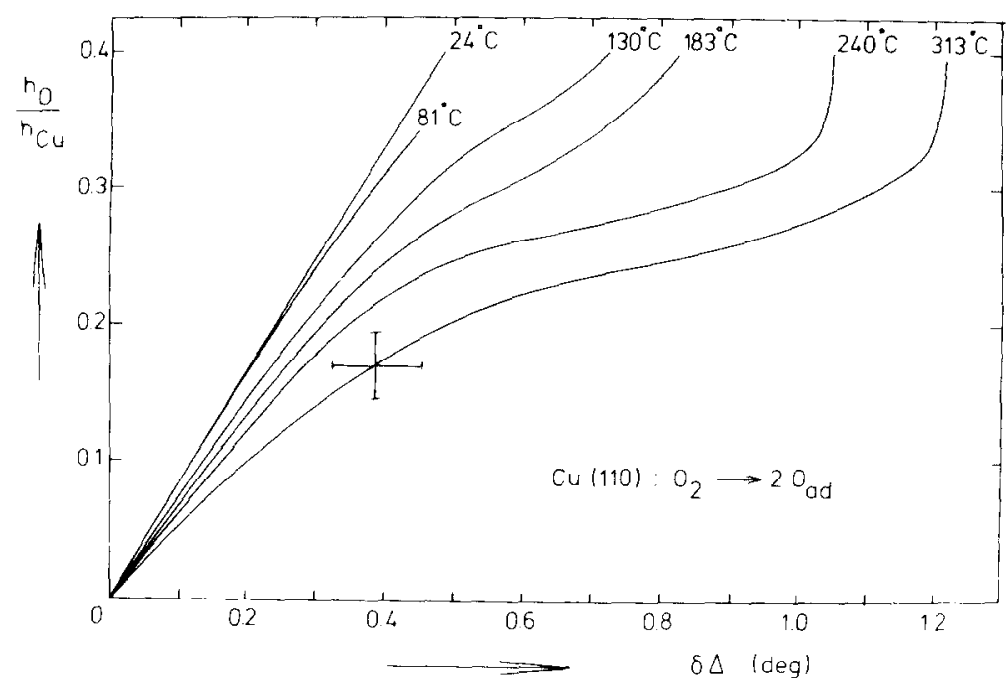

Fig. 9. Ratio of the $\mathrm{O} 510 \mathrm{eV}$ to the $\mathrm{Cu} 920 \mathrm{eV}$ Auger peak versus $\delta \Delta$ upon adsorption of oxygen on $\mathrm{Cu}(110)$ at different temperatures $\left(\Omega=90^{\circ}\right)$. From ref. [66].

of the oxygen Auger signal for several temperatures. Assuming the Auger signal to be proportional to the surface coverage, it follows that the $\delta \Delta$ per adsorbed atom is a function of $\Omega, T$ and coverage. $\delta \psi$ is only a function of coverage and $\Omega$ in the temperature range $24-313^{\circ} \mathrm{C}$; at higher temperatures $\left(450^{\circ} \mathrm{C}\right)$ and $\Omega=90^{\circ}, \delta \Delta$ decreases to $0.9^{\circ}$ and $\delta \psi$ increases to $0.25^{\circ}$. It thus appears that $\delta \Delta$ and $\delta \psi$ originate from different causes as discussed in section 4.2 , model $\mathrm{c}$. Within the framework of this model, the increase of $\delta \Delta$ with $T$ is assigned to an increased charge transfer to the oxygen atom. This is in accord with the increase in the change of workfunction with temperature, observed in the $\mathrm{Cu}(110) / 0$ system [69]. The constancy of $\delta \psi$ with temperature between $24-313^{\circ} \mathrm{C}$ then indicates a temperature-independent collision time $\tau$. This $\tau$ appears different for the different directions, due to the anisotropy of the (110) plane itself and/or to the anisotropic distribution of the chemisorbed oxygen atoms [66].

The decreased value of $\delta \Delta$ observed at $450^{\circ} \mathrm{C}$, combined with the observed increase in $\delta \psi$ might be related to the effect shown in fig. 5 , which indicates that increased charge transfer leads to a decrease in $\delta \Delta$ with a simultaneous increase in $\delta \psi$ around $\delta N_{\mathrm{f}} / N_{\mathrm{f}} \simeq 0.55$.

Some qualitative insight into the factors that determine the degree of anisotropy can be gained from calculations with model a with an anisotropic layer. For a copper substrate, covered with a $5 \AA$ layer of material with constant $\epsilon_{z}$ and variable $\epsilon_{x}$ and $\epsilon_{y}$ the following trends emerge:

(i) for constant $\epsilon_{x}^{\prime \prime}=\epsilon_{y}^{\prime \prime}, \delta \Delta$ increases with increasing $\epsilon^{\prime}$ in the plane of incidence, 
but decreases with $\epsilon^{\prime}$ perpendicular to the plane of incidence. These changes are such that upon rotating the surface $90^{\circ}$ along its normal the largest value of $\delta \Delta$ is obtained with the largest $\epsilon^{\prime}$ in the plane of incidence;

(ii) for constant $\epsilon_{x}^{\prime}=\epsilon_{y}^{\prime}, \delta \Delta$ is virtually independent of $\epsilon^{\prime \prime}$ perpendicular to, or in the plane of incidence and thus independent of crystal orientation;

(iii) for constant $\epsilon_{x}^{\prime \prime}=\epsilon_{y}^{\prime \prime}, \delta \psi$ is rather constant and small for small values of $k$, as can be expected;

(iv) for constant $\epsilon_{x}^{\prime}=\epsilon_{y}^{\prime}, \delta \psi$ increases with $\epsilon^{\prime \prime}$ in the plane of incidence and decreases with $\epsilon^{\prime \prime}$ perpendicular to the plane of incidence. The largest $\delta \psi$ is obtained with the largest $\epsilon^{\prime \prime}$ in the plane of incidence.

The same trends are obtained in a simpler way with Strachan's approach (section $2.4)$. For an absorbing dipole layer on copper $\left(\phi_{0}=69^{\circ}, \lambda=6328 \AA, \widetilde{n}_{2}=0.13\right.$ - i3.55), eqs. (22) and (23) yield

$$
\begin{aligned}
& \delta \Delta=2.10 \sigma_{z}^{\prime}+0.20 \sigma_{x}^{\prime}-0.038 \sigma_{y}^{\prime}+0.065 \sigma_{z}^{\prime \prime}-0.010 \sigma_{x}^{\prime \prime}+0.003 \sigma_{y}^{\prime \prime}, \\
& \delta \psi=-0.032 \sigma_{z}^{\prime}+0.005 \sigma_{x}^{\prime}-0.001 \sigma_{y}^{\prime}+1.05 \sigma_{z}^{\prime \prime}+0.10 \sigma_{x}^{\prime \prime}-0.019 \sigma_{y}^{\prime \prime} .
\end{aligned}
$$

From the values of the coefficients it follows that differences in $\delta \psi$ are primarily associated with difference in $\sigma_{j}^{\prime \prime}$ and differences in $\delta \Delta$ mainly with differences in $\sigma_{j}^{\prime}$. The observed positive values of $\delta \psi$ may be explained with complex scattering indices, e.g. a positive values of $\sigma_{z}^{\prime \prime}$. To explain the difference in $\delta \psi$ for $\mathrm{Cu}(110)$ at $\Omega=0^{\circ}$ and $90^{\circ}$ one has to assume that along the rows ([110] direction) $\sigma^{\prime \prime}\left(\alpha^{\prime \prime}\right)$ is much larger than it is perpendicular to the rows ([001]). The difference in $\delta \Delta$ at higher temperatures (fig. 8) may be ascribed to a much larger value of $\sigma^{\prime}\left(\alpha^{\prime}\right)$ perpendicular to the rows.

The equality in trends, derived with both approaches, should be expected, because usually in good approximation $\epsilon \simeq 1+4 \pi N \widetilde{\alpha}$, and thus, with eq. (24), $\epsilon_{j}^{\prime} \simeq$ $1+$ const. $X \sigma_{j}^{\prime}$ and $\epsilon_{j}^{\prime \prime} \simeq$ const. $X \sigma_{j}^{\prime \prime}$.

An interpretation in terms of chemical bonding and charge distribution is impracticable at the moment. Final conclusions will be drawn after measurements of the oxygen chemisorption at different wavelengths and comparison with results to be obtained with other techniques, e.g. UPS.

\section{Acknowledgements}

The investigations were supported by the Netherlands Foundation for Chemical Research (SON) with financial aid from the Netherlands Organization for the Advancement of Pure Research (ZWO). 
Table 6

Optical data for $\mathrm{Cu}$ and $\mathrm{Ag}, \lambda=6328 \AA$

\begin{tabular}{lllllllll}
\hline$\epsilon^{\prime}$ & $\epsilon^{\prime \prime}$ & $\begin{array}{l}\epsilon_{\mathrm{b}}^{\prime} \\
\text { ref. } \\
{[83]}\end{array}$ & $\begin{array}{l}m^{*} / m \\
\text { ref. } \\
{[83]}\end{array}$ & $N_{\mathrm{f}}\left(\mathrm{cm}^{-3}\right)$ & $\begin{array}{l}\sigma_{0}^{*}\left(\mathrm{~s}^{-1}\right) \\
\text { ref. }[84]\end{array}$ & $\tau(\mathrm{s})$ & $\epsilon_{\mathrm{b}}^{\prime \prime}$ \\
\hline $\mathrm{Cu}$ & -12.6 & 0.92 & 7.5 & 1.42 & $8.3 \times 10^{22}$ & $3.6 \times 10^{17}$ & $2.4 \times 10^{-14}$ & 0.63 \\
$\mathrm{Ag}$ & -20.6 & 0.49 & 2.5 & 0.95 & $6.1 \times 10^{22}$ & $4.2 \times 10^{17}$ & $2.6 \times 10^{-14}$ & 0.13 \\
\hline
\end{tabular}

\section{Appendix}

In the classical treatment of the dielectric constant of a metal, $\epsilon=\epsilon^{\prime}-\mathbf{i} \epsilon^{\prime \prime}$, two contributions are distinguished, that of free and of bound electrons [82]:

$\epsilon^{\prime}=\epsilon_{\mathrm{f}}^{\prime}+\epsilon_{\mathrm{b}}^{\prime}=1-\nu_{1}^{2} /\left(\nu^{2}+\nu_{2}^{2}\right)+\epsilon_{\mathrm{b}}^{\prime}$,

$\epsilon^{\prime \prime}=\epsilon_{\mathrm{f}}^{\prime \prime}+\epsilon_{\mathrm{b}}^{\prime \prime}-\left(\nu_{2} / \nu\right) \nu_{1}^{2} /\left(\nu^{2}+\nu_{2}^{2}\right)+\epsilon_{\mathrm{b}}^{\prime \prime}$.

Here $\nu$ is the frequency of the light, $\nu_{1}$ the plasma frequency and $\nu_{2}$ the electron collision frequency. The plasma frequency is related to the density $N_{\mathrm{f}}$, the optical mass $m^{*}$ and the charge $e$ of the free electrons:

$\nu_{1}^{2}=e^{2} N_{\mathrm{f}} / \pi m^{*}$.

Further,

$\nu_{2}=1 / 2 \pi \tau=\nu_{1}^{2} / 2 \sigma_{0}^{*}$,

where $\tau$ is the collision time and $\sigma_{0}^{*}$ the conductivity at visible frequencies. Neglecting $\nu_{2}^{2}$ with respect to $\nu^{2}$, we obtain

$\epsilon^{\prime}=n^{2}-k^{2}=1-e^{2} N_{\mathrm{f}} / \pi m^{*} \nu^{2}+\epsilon_{\mathrm{b}}^{\prime}$,

$\epsilon^{\prime \prime}=2 n k=e^{2} N_{\mathrm{f}} / 2 \pi^{2} m^{*} \pi \nu^{3}+\epsilon_{\mathrm{b}}^{\prime \prime}$.

A summary of the optical data for bulk $\mathrm{Cu}$ and $\mathrm{Ag}$ is given in table 6. At $\lambda=$ $6328 \AA$, the values of $\epsilon^{\prime}$ and $\epsilon^{\prime \prime}$ were taken from ref. [34] for $\mathrm{Cu}$ and from ref. [35] for $\mathrm{Ag}$ (see table 1). Using literature values for $\epsilon_{\mathrm{b}}^{\prime}, \mathrm{m}^{*}$ and $\sigma_{0}^{*}$, we calculated $N_{\mathrm{f}}$ with eq. (A.5), $\tau$ with eqs. (A.3) and (A.4), and finally $\epsilon_{\mathrm{h}}^{\prime \prime}$ with eq. (A.6).

\section{References}

[1] P. Drude, Ann. Physik. Chem. 36 (1889) 532, 865; 39 (1890) 481.

[2] Lord Rayleigh, Phil. Mag. 33 (1892) 1.

[3] For historical review see: A. Rothen, in: Ellipsometry in the Measurement of Surfaces and 
Thin Films, Eds. E. Passaglia, R.R. Stromberg and J. Kruger (Natl. Bur. Std. (US) Misc. Publ. 256, Washington, DC, 1964) p. 201.

[4] L. Tronstad, Trans. Faraday Soc. 29 (1933) 502.

[5] R.J. Archer and G.W. Gobeli, J. Phys. Chem. Solids 26 (1965) 343.

[6] G.A. Bootsma and F. Meyer, Surface Sci. 14 (1969) 52.

[7] F. Meyer, Surface Sci. 56 (1976) 37.

[8] F. Meyer, F.F. de Kluizenaar and G.A. Bontsma, Surface Sci. 27 (1971) 88.

[9] G. Quentel, J.M. Rickard and R. Kern, Surface Sci. 50 (1975) 343.

[10] G. Quentel and R. Kern, Surface Sci. 55 (1976) 545.

[11] C. Strachan, Proc. Cambridge Phil. Soc. 29 (1933) 116.

[12] D.V. Sivukhin, Zh. Eksperim. i Teor. Fiz. SSSR 18 (1948) 976; 21 (1951) 367.

[13] D.V. Sivukhin, Soviet Phys.-JETP 3 (1956) 269.

[14] P.W. Atkins and A.D. Wilson, Surface Sci. 22 (1970) 433.

[15] P.W. Atkins and A.D. Wilson, in: Solid State Surface Sci., Vol. 2, Fd. M. Green (Dekker, New York, 1973) p. 153.

[16] A.J. Bennett and D. Penn, Phys. Rev. B11 (1975) 3644.

[17] A.A. Maradudin, Surface Sci. 81 (1979) 13.

[18] W.J. Plieth and K. Naegele, Surface Sci. 64 (1977) 484.

[19] W.J. Plieth, J. Physique 38 (1977) C5-215.

[20] H. Schopper, Z. Physik 132 (1952) 146.

[21] R.M.A. Azzam and N.M. Bashara, Ellipsometry and Polarized Light (North-Holland, Amsterdam, 1977) p. 357.

[22] D. den Engelsen, J. Opt. Soc. Am. 61 (1971) 1460.

[23] A.W. Adamson, Physical Chemistry of Surfaces, 3rd ed. (Wiley, New York, 1976) ch. 2.

[24] J.D.E. McIntyre, in: Optical Properties of Solids; New Developments, Ed. B.O. Seraphin (North-Holland, Amsterdam, 1976) p. 555.

[25] C.J.F. Böttcher, Theory of Electric Polarization, 2nd ed., Vol. I (Elsevier, Amsterdam, 1973).

[26] C. Kittel, Introduction to Solid State Physics, 5th ed. (Wiley, New York, 1976) ch. 13.

[27] D. den Engelsen, Surface Sci. 56 (1976) 272.

[28] J.A. Osborn, Phys. Rev. 67 (1945) 351.

[29] E.C. Stoner, Phil. Mag. 36 (1945) 803.

[30] Table 1 of ref. [6] contains a printing error: for the Strachan model $M=4 \pi \sigma_{x}$.

[31] M.J. Dignam and M. Moskovits, J. Chem. Soc. Faraday II, 69 (1973) 56.

[32] M.J. Dignam and J. Fedyk, J, Physique 38 (1977) C5-57.

[33] G.D. Mahan and A.A. Lucas, J. Chem. Phys. 68 (1978) 1344.

[34] F.H.P.M. Habraken, E.P. Kieffer and G.A. Bootsma, in: Proc. 7th Intern. Vacuum Congr. and 3rd Intern. Conf. on Solid Surfaces (Vienna, 1977) p. 877.

[35] H. Albers, J.M.M. Droog and G.A. Bootsma, Surface Sci. 64 (1977) 1.

[36] F.C. Schouten, E.W. Kaleveld and G.A. Bootsma, Surface Sci. 63 (1977) 460.

[37] F. Meyer and M.J. Sparnaay, in: Surface Physics of Phosphors and Semiconductors, Eds. C.G. Scott and C.E. Reed (Academic Press, London, 1975) p. 391.

[38] Recent reviews: M.A. Van Hove, in: The Nature of the Surface Chemical Bond, Eds. T.N. Rhodin and G. Ertl (North-Holland, Amsterdam, 1979); M.A. Van Hove, Surface Sci. $80(1979) 1$.

[39] M. Alff and W. Moritz, Surface Sci. 80 (1979) 24.

[40] H.L. Davis, J.R. Noonan and L.H. Jenkins, Surface Sci. 83 (1979) 559.

[41] J.E. Demuth, P.M. Marcus and D.W. Jepsen, Phys. Rev. B11 (1975) 1460.

[42] J.F. van der Veen, R.G. Smeenk, R.M. Tromp and F.W. Saris, Surface. Sci. 79 (1979) 212.

[43] J.C. Maxwell Garnett, Phil. Trans. Roy. Soc. (London) 203 (1904) 385; 205 (1906) 237; O.S. Heavens, Optical Properties of Thin Solid Films (Dover, New York, 1965) p. 177. 
[44] W.E.J. Neal, Appl. Surface Sci. 2 (1979) 445.

[45] O. Hunderi, Surface Sci. 96 (1980) 1; O. Hunderi, J. Physique 38 (1977) C5-89.

[46] P.B. Johnson and R.W. Christy, Phys. Rev. B6 (1972) 4370.

[47] J.M.M. Droog, private communication.

[48] J.M.M. Droog, P.T. Alderliesten and G.A. Bootsma, J. Electroanal. Chem. 99 (1979) 173.

[49] 7.I. Kudryavtseva, N.A. Shumilova, V.A. Openkin, N.A. Zhuchkova and I.I. Khruscheva, Soviet Electrochem. 13 (1977) 608.

[50] M.Ph. Stoll, J. Appl. Phys. 40 (1969) 4533.

[51] N.J. Chou, J.M. Eldridge, R. Hammer and D. Dong, J. Electron. Mater. 2 (1973) 115.

[52] J.J. Carroll and A.J. Melmed, Surface Sci. 16 (1969) 251.

[53] A.J. Melmed and J.J. Carroll, J. Vacuum Sci. Technol. 10 (1973) 164.

[54] U. Merkt, S. Ritz and P. Wissmann, in: Proc. 7th Intern. Vacuum Congr. and 3rd Intern. Conf. on Solid Surfaces (Vienna, 1977) p. 1801.

[55] U. Merkt and P. Wissmann, Thin Solid Films 57 (1979) 65.

[56] R.C. O'Handley and D.K. Burge, Surface Sci. 48 (1975) 214.

[57] G.W. Rubloff, J. Anderson and P.J. Stiles, Surface Sci. 37 (1973) 75.

[58] G.W. Rubloff, J. Anderson, M.A. Passler and P.J. Stiles, Phys. Rev. Letters 32 (1974) 667.

[59] M. Watanabe and A. Hirakuta, Japan. J. Appl. Phys. 18 (1979) 31.

[60] M.J. Dignam and M. Moskovits, J. Chem. Soc. Faraday II 69 (1973) 65.

[61] M.J. Dignam, B. Rao and J. Roth, Trans. Faraday Soc. 69 (1973) 804.

[62] M. Moskovits and P. McBreen, J. Chem. Phys. 68 (1978) 4992.

[63] W.H. Wyrobisch, Thesis, Technical Univ. Munich (1979).

[64] H. Albers, W.J.J. van der Wal, O.L.J. Gijzeman and G.A. Bootsma, Surface Sci. 77 (1978) 1.

[65] F.H.P.M. Habraken, E.Ph. Kieffer and G.A. Bootsma, Surface Sci. 83 (1979) 45.

[66] F.H.P.M. Habraken and G.A. Bootsma, Surface Sci. 87 (1979) 333.

[67] F.H.P.M. Habraken, C.M.A.M. Mesters and G.A. Buotsnla, to be published.

[68] P.K. de Bokx, F. Labohm, O.L.J. Gijzeman, G.A. Bootsma and J.W. Geus, Appl. Surface Sci., in press.

[69] F.H.P.M. Habraken, G.A. Bootsma, P. Hofmann, S. Hachicha and A.M. Bradshaw, Surface Sci. 88 (1979) 285.

[70] F.L. McCrackin, A. Fortran Program for Analysis of Ellipsometer Measurements (Natl. Bur. Std. (US) Tech. Note 479, Washington, DC, 1969).

[71] J.V. Catheart and G.F. Petersen, in: Ellipsometry in the Measurement of Surfaces and Thin Films, Eds. E. Passaglia, R.R. Stromberg and J. Kruger (Natl. Bur. Std. (US) Misc. Publ. 256, Washington, DC, 1964) p. 201.

[72] E.C. Butcher, A.J. Dyer and N.E. Gilbert, Brit. J. Appl. Phys. 1 (1968) 1673.

[73] J. Spyridelis, J. Stoimenos and N. Economou, Phys. Status Solidi 20 (1967) 623.

[74] R.J. Powell and W.E. Spicer, Phys. Rev. B2 (1970) 2183.

[75] J.D.E. McIntyre, in: Advances in Electrochemistry and Electrochemical Engineering, Vol. 9, Ed. R.H. Muller (Wiley, New York, 1973) p. 61.

[76] J.W. Geus, in: Chemisorption and Reactions on Metallic Films, Ed. C.J.R. Anderson (Academic Press, London, 1971) Vol. 1, ch. 5, and references therein.

[77] T.E. Furtak and D.W. Lynch, Phys. Rev. Letters 35 (1975) 960.

[78] R. Kofmann, P. Cheyssac and J. Richard, Surface Sci. 77 (1978) 537.

[79] J.V. Cathcart, J.E. Epperson and G.V. Petersen, Acta Met. 10 (1962) 699.

[80] B.S. Borie, C.J. Sparks and J.V. Cathcart, Acta Met. 10 (1962) 691.

[81] D.M. Kolb, R. Kötz and K. Yamamoto, Surface Sci. 87 (1979) 20.

[82] R.E. Hummel, Optische Eigenschaften von Metallen und Legierungen (Springer, Berlin, 1971). 
[83] H. Ehrenreich and H.R. Philipp, Phys. Rev. 128 (1962) 1622.

[84] M. Otter, Z. Physik 161 (1961) 163.

\section{Discussion}

O. Hunderi (University of Trondheim): The interpretation of data in this wavelength range is complicated by the possibility of electron transitions between bonding or antibonding states and conduction band states in the metal. It would therefore be best to go to the near IR so that you are below the onset of such transitions, then the models presented would be more relevant.

G.A. Bootsma: I agree. In fact we intend to perform these measurements in the near future.

D.E. Aspnes (Bell Laboratories): The microscopic models that you mention are basically efforts to calculate the local fields at the sites of point polarizable particles on a surface or a regular array of atoms. But unless there is virtually no interaction between substrate and adsorbate (weak physisorption) these models are also phenomenological and cannot be used in a first-principles calculation of the effects of adsorbed layers on optical response, as your data show. These models should therefore be considered to be equivalent to the purely phenomenological macroscopic description in terms of a thin film with a model thickness and dielectric function.

G.A. Bootsma: We do not feel that the models reviewed by us are completely equivalent to a thin film plus dielectric constant approximation. Rather, the approaches try to calculate the film dielectric function from "known" properties of the constituents. The big problem is of course to what extent the polarizability of, e.g., an oxygen atom is independent of its environment (free, or chemisorbed on a metal) and how much the dielectric constant of the bare metal selvedge changes upon chemisorption. Instead of lumping all these unknown quantities into one "effective dielectric constant" we have tried to model the expected changes in the dielectric constant by admittedly rough and possibly inadequate assumptions (see in particular model c).

J.B. Theeten (Lab. d'Electronique et de Physique Appliqueé): Your results on the anisotropy of $\delta \Delta$ versus azimuth for the $\mathrm{O}_{2}$ adsorption on $\mathrm{Cu}(110)$ are very interesting and a correlation of these with angular resolved ultra violet photoemission would be very informative.

G.A. Bootsma: Yes, I completely agree that especially a comparison with angular resolved UPS data taken at different temperatures would be fruitful to explain the increase of $\delta \Delta$ at equal oxygen coverages with increasing temperature and to check the applicability of model c.

T.H. Allen (Optical Coating Lab.): (1) Did you observe in the measured $\delta \Delta$ and $\delta \psi$ values during the ageing of the metal surfaces any evidence for a transition from physically to chemically adsorbed oxygen. If so, did you detect any chemical shifts in either the metal or oxygen Auger peaks? (2) Did you measure the mass distribution of residual gases in the vacuum chamber?

G.A. Bootsma: We have never found any evidence for a transition from physically to chemically adsorbed oxygen in our measured $\delta \Delta$ and $\delta \psi$ values. From an analysis of the kinetic data of the chemisorption of oxygen on copper (see ref. [67]) we got indications that such a transition may indeed play a role (precursor state model). However, at the pressures and temperatures of our study the coverage of physically adsorbed oxygen is expected to be $\$ 10^{-4}$ monolayer. According to table 1 the change in $\Delta$ will be too small $\left(\sim 10^{-4} \mathrm{deg}\right)$ to be detected. Qualitatively the same holds for the most important residual gases $\left(\mathrm{CO}, \mathrm{CO}_{2}\right)$ in our vacuum system $\left(p<10^{-9}\right.$ Torr). Thus it is not surprising that we have never detected an Auger shift associated with the transition from physically to chemically adsorbed oxygen. It may be remarked that a shift in the O(KLL) Auger peak has been reported by Benndorf et al. (J. Phys. Chem. Solids 40 (1979) 877) for a transition from one into another chemisorption state of oxygen on $\mathrm{Cu}(100)$. 